\title{
Sustained attention in skilled and novice martial arts athletes: A study of Event-Related Potentials and Current Sources
}

Javier Sanchez-Lopez, Juan Silva-Pereyra, Thalia Fernandez

Background. Research on sports has revealed that behavioral responses and eventrelated brain potentials (ERP) are better in expert than in novice athletes for sport-related tasks. Focused attention is essential for optimal athletic performance across different sports but mainly in combat disciplines. During combat, long periods of focused attention (i.e., sustained attention) are required for a good performance. Few investigations have reported effects of expertise on brain electrical activity and its neural generators during sport-unrelated attention tasks. The aim of the present study was to assess the effect of expertise (i.e., skilled and novice martial arts athletes) analyzing the ERP during a sustained attention task (Continuous Performance Task; CPT) and the cortical threedimensional distribution of current density, using the sLORETA technique. Methods. CPT consisted in an oddball-type paradigm presentation of five stimuli (different pointing arrows) where only one of them (an arrow pointing up right) required a motor response (i.e., target). CPT was administered to skilled and novice martial arts athletes while EEG were recorded. Amplitude ERP data from target and non-target stimuli were compared between groups. Subsequently, current source analysis for each ERP component was performed on each subject. SLORETA images were compared by condition and group using Statistical Non-Parametric Mapping analysis. Results. Skilled athletes showed significant amplitude differences between target and non-target conditions in early ERP components (P100 and P200) as opposed to the novice group; however, skilled athletes showed no significant effect of condition in N200 but novices did show a significant effect. Current source analysis showed greater differences in activations in skilled compared with novice athletes between conditions in the frontal (mainly in the Superior Frontal Gyrus and Medial Frontal Gyrus) and limbic (mainly in the Anterior Cingulate Gyrus) lobes. Discussion. These results are supported by previous findings regarding activation of neural structures that underlie sustained attention. Our findings may indicate a better-controlled attention in skilled athletes, which suggests that expertise can improve effectiveness in allocation of attentional resources during the first stages of cognitive processing during combat. 


\section{Sustained Attention in Skilled and Novice}

\section{Martial Arts Athletes: A Study of Event-}

\section{Related Potentials and Current Sources}

4 Javier Sánchez-López ${ }^{1}$, Juan Silva-Pereyra² ${ }^{2}$, Thalía Fernández ${ }^{1}$

5 Laboratorio de Psicofisiología, Departamento de Neurobiología Conductual y Cognitiva, Instituto de

6 Neurobiología, UNAM Campus Juriquilla, Querétaro, México.

$7 \quad$ 2Unidad de Investigación Interdisciplinaria en Ciencias de la Salud y la Educación (UIICSE), Facultad de

8 Estudios Superiores Iztacala, UNAM, Los Reyes Iztacala, Tlalnepantla, Estado de México, México.

12 Corresponding Author:

13 Thalía Fernández ${ }^{1}$

14 Boulevard Juriquilla 3001, Querétaro, 76230, México

15 E-mail address: thaliafh@yahoo.com.mx 


\section{Abstract}

19 Background. Research on sports has revealed that behavioral responses and event-related brain potentials (ERP) are better in expert than in novice athletes for sport-related tasks. Focused attention is essential for optimal athletic performance across different sports but mainly in combat disciplines. During combat, long periods of focused attention (i.e., sustained attention) are required for a good performance. Few investigations have reported effects of expertise on brain electrical activity and its neural generators during sport-unrelated attention tasks. The aim of the present study was to assess the effect of expertise (i.e., skilled and novice martial arts athletes) analyzing the ERP during a sustained attention task (Continuous Performance Task; CPT) and the cortical three-dimensional distribution of current density, using the sLORETA technique.

Methods. CPT consisted in an oddball-type paradigm presentation of five stimuli (different pointing arrows) where only one of them (an arrow pointing up right) required a motor response (i.e., target). CPT was administered to skilled and novice martial arts athletes while EEG were recorded. Amplitude ERP data from target and non-target stimuli were compared between groups. Subsequently, current source analysis for each ERP component was performed on each subject. sLORETA images were compared by condition and group using Statistical NonParametric Mapping analysis.

Results. Skilled athletes showed significant amplitude differences between target and non-target conditions in early ERP components (P100 and P200) as opposed to the novice group; however, skilled athletes showed no significant effect of condition in N200 but novices did show a 
39 significant effect. Current source analysis showed greater differences in activations in skilled

40 compared with novice athletes between conditions in the frontal (mainly in the Superior Frontal

41 Gyrus and Medial Frontal Gyrus) and limbic (mainly in the Anterior Cingulate Gyrus) lobes.

42 Discussion. These results are supported by previous findings regarding activation of neural

43 structures that underlie sustained attention. Our findings may indicate a better-controlled

44 attention in skilled athletes, which suggests that expertise can improve effectiveness in allocation

45 of attentional resources during the first stages of cognitive processing during combat. 
${ }_{47}$ Introduction

Sports performance and training encompass the development of physical, technical-tactical, and psychological skills. Among the psychological abilities, sport training enhances emotional and cognitive aspects. Cognitive processes are essential for optimal sports performance, and attention-related processes are particularly important in combat sports (Anshel \& Payne 2006; Blumenstaein et al. 2002; del-Monte 2005; Lavalle et al. 2004; Rushall 2006; Sánchez-López et al. 2013; Sánchez-López et al. 2014). Previous studies have reported the outstanding attentional capacities of sport experts, who can also more quickly extract and identify the most important and relevant information (Abernethy \& Russell 1987; del-Monte 2005; Sánchez-López et al. 2014; Williams \& Grant 1999). Thus, skilled athletes can better modulate their attention resources according to specific environmental requirements (Nougier \& Rossi 1999). Integrative mind-body training, such as meditation, martial arts, and yoga, is known to enhance brain and cognitive functions, specifically attentional processes (Brefczynski-Lewis et al. 2007; Tang \& Posner 2009). Focused attention is essential for open-skill sports such as team sports and combat. Since, in combat sports, long periods of focused attention are required during competition, it could be one of the most relevant processes for high performance, and one movement attended or missed can lead to victory or failure, respectively; however, it remains unclear whether a kind of attention related to maintaining focus (i.e., sustained attention) is the key to the performance of experts in these disciplines. Based on this idea, the aim of this study was to evaluate sustained attention in martial arts disciplines.

One method of understanding how sports performance is enhanced is by studying brain electrical activity through the event-related potentials (ERP) technique (Thompson et al. 2008). 
ERP, which are regarded as temporal correlates of information processing (Jennings \& Coles 1991; Picton et al. 2000), allow us to understand the temporal dynamics of the different subprocesses of a global cognitive aspect such as attention. However, given that attention models involve several brain areas that interact in different ways with every attention subprocess, current source analysis is necessary. The standardized low-resolution brain electromagnetic tomography (sLORETA) is a suitable method to precisely locate brain electrical source. The role of expertise and training in attention and brain activity has been investigated using the ERP technique and sLORETA. In previous studies (Babiloni et al. 2010a; Del Percio et al. 2010; Fontani \& Lodi 2002; Fontani et al. 2006; Fontani et al. 1999; Hack et al. 2009; Hamon \& Seri 1989; Hung et al. 2004; Radlo et al. 2001), behavioral performance, electrophysiological brain activity, and current sources were shown to have distinct characteristics when compared between experts and non-experts or non-athletes, which suggests that people with training in different skills and sports may have attentional profiles related to their expertise. Specifically, ERP studies have found larger amplitudes in components associated with attention (e.g., P100, P200, and P300) in expert athletes than in other populations (Hack et al. 2009; Hamon \& Seri 1989; Hung et al. 2004; Jin et al. 2011; Ozmerdivenli et al. 2005; Zwierko et al. 2011); the authors interpreted these higher amplitudes as indicators of better attentional mechanisms in experts. Few studies have performed current source analysis to investigate cognitive differences between expert and novice athletes. By using sLORETA, Del Percio et al. (2010) studied differences in activation of the premotor and motor brain areas during hand movements between karate athletes and non-athletes. Their results showed less activation of these structures in athletes as compared to activation in non-athletes. Babiloni et al. (2010b) found differences in the activation of the dorsal and frontoparietal "mirror" pathways between expert, non-expert 
92 athletes and non-athletes. Both studies supported the "Neural Efficiency" hypothesis. This

93 hypothesis proposes that efficiency would be observed as spatial cortical reduction of the task-

94 related brain activity in expert athletes when compared with less expert groups (Babiloni et al.

95 2009). However, these studies have not investigated the effect of expertise by analyzing the

96 current sources of electrical brain activity in athletes during any attentional task.

97 Several ERP studies have investigated the neural correlates of sustained attention by

98 using the continuous performance task and their results have shown different waves that mirror

99 brain electrical modulations to specific demands of attention; among the main ERP components

100 reported in the literature, P100, N100, P200, N200 and P300 are found (for review, see Riccio et

101 al. 2002). Although there are no studies that specifically report what areas in the brain are related

102 to sustained attention in athletes, evidence from lesion and functional imaging studies shows that

103 some brain areas, such as the anterior cingulate and dorsolateral prefrontal as well as parietal

104 cortical regions primarily in the right hemisphere, are consistently activated in participants who

105 performed sustained attention tasks (Cohen et al. 1992; Fink et al. 1997; Pardo et al. 1991).

106 Previous studies using neuroimaging techniques describe sustained attention as a top-down

107 mechanism that begins with the motor and cognitive readiness for the subject to detect and

108 discriminate the stimulus information; this process is mediated by right fronto-parietal brain

109 areas, and it facilitates perceptual and spatial attentional processes that contribute to the

110 performance by recruiting parietal areas related to sensory processing (Hopfinger et al. 2000;

111 Lane et al. 1999). The perceptual facilitation of attentional processes via top-down mechanisms

112 could result in increased firing activity in neurons that response selectively in sensory-

113 association areas when attentional task demands are increased (Desimone 1996). 
115 focus throughout the competition, i.e., sustained attention, which implies maintaining attentional

116 focus for long periods of time. This is an essential attentional component that prepares the

117 subject to detect unpredictable stimuli over prolonged time periods (Sarter et al. 2001).

118 Considering the extensive literature about the uses and efficiency of the continuous performance

119 task (CPT) (Smid et al. 2006), we propose the use of this task in a classical version for the study

120 of sustained attention in martial arts athletes, i.e. sport un-related task in order to avoid

121 advantages related to the sport features in skilled athletes. Prior to a sustained attention task,

122 subjects are instructed to attend to the same specific target stimulus in the presence of other non-

123 target stimuli. Every stimulus represents a potential target that may require a response.

124 Considering that combat requires long periods of sustained attention and that this ability

125 should be better developed in skilled athletes, our hypothesis, in accord with previous studies, is

126 that skilled athletes would show a better performance as reflected in larger amplitudes and

127 shorter latencies in the principal ERP components associated with attention, as compared to

novice athletes. This difference between groups should also be detectable as prolonged,

extended-focus activations in the sustained-attention-related brain regions (i.e., anterior

cingulate, prefrontal and parietal areas). We propose that skilled athletes will show better

attentional abilities, which will be reflected as better performance in the sustained-attention task,

and that this performance can be related to differences in the various components of the ERP

133 (particularly larger amplitudes in the components related to attention: P100, P200 and P300),

134 consistent with previous reports (Hack et al. 2009; Hamon \& Seri 1989; Hung et al. 2004; Jin et

136 features will likely correlate with greater activation, in skilled athletes, in the brain structures 
137 implicated in sustained attention (anterior cingulate, dorsolateral prefrontal, and parietal cortical 138 regions primarily in the right hemisphere) as previous neuropsychological studies report (Cohen 139 et al. 1992; Fink et al. 1997; Pardo et al. 1991). Therefore, this study aims to evaluate the

140 differences in sustained attention between skilled and novice martial arts athletes using ERP and 141 sLORETA as tools. 


\section{Materials and Methods}

144

145

146

147

148

149

150

151

152

153

154

155

156

157

158

159

160

161

162

163

\section{Participants}

In order to evaluate sustained attention related to sport expertise in martial arts disciplines, we recruited twenty-one martial arts athletes from judo, tae-kwon-do, and kung-fu disciplines. Degree of combat rank in martial arts is mainly defined by the level of the combat martial training, which includes knowledge and application of the techniques, psychology, and philosophy of the martial arts discipline to real combat. These abilities are assessed with the completion of a theoretical and practical exam and the subsequent delivery of the degree (e.g. a belt with a specific rank). Considering the afore mentioned variables, two groups of athletes were formed: a) 11 skilled athletes (mean age $=25.4$ years, $\mathrm{SD}=11.5$ ) holding the highest combat degree (i.e. black belt or the highest in each discipline), or/and at least five years of sport practice, a report about the athlete's expertise from the team coach, and competitive experience in national and international competitions. b) 10 novice athletes (mean age $=25.5$ years, $\mathrm{SD}=9$ ) with the lowest combat degree (i.e. no belt or white belt in their discipline), less than one year of sport practice, a report about the athlete's newness from the team coach, and with no competitive experience. All participants were right handed and healthy, with vision that was normal or corrected to normal. All participants showed scores in the normal range $(>90)$ on the Wechsler Intelligence Scale and in the task of variables of attention (TOVA) with a score greater than 1.80, indicating normal attention. No differences in age, intelligence, ADHD and educational level between groups were observed. Additionally, a mini-mental test (Folstein et al. 1975) and a neurological evaluation were conducted to confirm no neurological disorders. Before the EEG 
164 recording, participants were asked about medication and beverage consumption that could 165 possibly influence the attentional status (coffee and alcohol intake, stimulating drinks, etc.).

166 Athletes who consume or had consumed medications or drugs that affect the nervous system in

167 the last year were eliminated from the study. EEG database of participants from a previous study

168 performed by the authors where motor-related cortical potentials were investigated (Sanchez-

169 Lopez et al. 2014) together with data of new participants were analyzed for this paper.

170 Participants were informed of their rights, and they provided written informed consent for

171 participation in the study. This research was carried out ethically and approved by the Ethics

172 Committee of the Instituto de Neurobiología at the Universidad Nacional Autónoma de México.

173 Summary of the characteristics of the participants are detailed in Table 1.

[Insert Table 1]

175

176

177

178

179

180

181

182

183

184

185

\section{Stimuli}

In this study, the stimuli used were white, 2.95-cm-wide, 2.03-cm-high, arrows pointed in five different directions. The random sequence of arrows was shown at the center of a 17-inch VGA computer monitor on a black background viewed from a distance of $80 \mathrm{~cm}$ and at a visual angle of $2.11 \times 1.451^{\circ}$.

\section{Continuous performance task}

The task consisted in the presentation of six blocks of 100 arrows each, to complete a sequence of 600 arrows shown to each participant. The subjects were asked to press a button as quickly as possible when the target arrow (pointed right and downward) appeared and not to respond when any other arrow was shown. The stimuli presented were $20 \%$ target and $80 \%$ nontarget. The stimulus duration was $100 \mathrm{~ms}$ with an inter-stimulus interval that varied between 
1861200 and $1500 \mathrm{~ms}$ and a response interval overlapped with the inter-stimulus interval (see Fig. 187 1).

$$
\text { [Insert Fig. 1] }
$$

\section{Procedure}

All participants were prepared with the electroencephalographic system and seated in a

191

192

193

\section{ERP Recording}

chair in a dimly lit room. Task instructions asking the subjects to press a button with their right hand as rapidly and accurately as possible when the target stimulus appeared were provided before the CPT performance.

$$
\text { During the CPT performance, an electroencephalogram (EEG) was recorded using }
$$

NeuroScan SynAmps amplifiers (Compumedics NeuroScan) and Scan 4.5 software

(Compumedics NeuroScan) with $32 \mathrm{Ag} / \mathrm{Cl}$ electrodes mounted on an elastic cap. Linked earlobes were used as references. Oculograms were also recorded from a supraorbital electrode, and an electrode was placed at the external canthus of the left eye. A 500-Hz sampling rate was used to digitalize the EEG with a band-pass filter set from 0.1 to $100 \mathrm{~Hz}$. Electrode impedances were maintained below $5 \mathrm{k} \Omega$.

\section{Data analysis}

\section{Behavioral Analysis}

Behavioral analysis was computed using percentages of correct responses, which were transformed [ARCSIN(Square Root (percentage/100))]. Data for the hit rates, false alarms, and response times were compared between groups (skilled and novices) using the two-sample t-test. 
207 ERP Analysis

208 The ERP were computed offline using $1200 \mathrm{~ms}$ epochs from each subject and

209 experimental condition (i.e., target and non-target). Each epoch consisted of the $200 \mathrm{~ms}$

210 preceding the stimulus and the $1000 \mathrm{~ms}$ following the presentation of stimulus. Epochs with

211 voltage changes exceeding $+80 \mu \mathrm{V}$ were automatically omitted from the final average.

212 Continuous EEG Segments were visually inspected and those with artifacts and electrical noise

213 were rejected. An eye-movement correction algorithm was applied to remove blinks and vertical

214 ocular-movement artifacts (Gratton et al. 1983). Low pass filtering for $40 \mathrm{~Hz}$ and $12 \mathrm{~dB}$ slope

215 was performed offline (Luck 2005). Further, a baseline correction was performed using the 200

216 ms pre-stimulus time window mentioned above. The averaged trials included only those with

217 correct responses.

218 Statistical analyses of amplitude and latency were separately performed using time

219 windows selected by visual inspection and maximum peak detection to select the time period of

220 all components observed. P100 (100-120 ms), P200 (190-210 ms), N200 (230-290 ms), and

221 P300 (350-500 ms) were the principal waves identified. A series of ANOVAs was also

222 separately performed for each ERP component (time window) and by considering lateral regions

223 or midline electrodes. In order to include the more possible electrodes in the analyses, 24

224 electrodes from left and right regions were analyzed with Group (skilled and novice) as between-

225 subject factor; Condition (target and non-target), Hemisphere (left and right) and Electrode site

226 (FP1, FP2, F3, F4, C3, C4, P3, P4, O1, O2, F7, F8, T3, T4, T5, T6, CP3, CP4, FC3, FC4, TP7,

227 TP8, FT7 and FT8) as within-subject factors were included. Other series of ANOVAs was

228 performed using midline electrodes. These analyses included Group (skilled and novices) as

229 between-subject factor, and Condition (target and non-target) and Electrode site (FZ, FCZ, CZ, 
$230 \mathrm{CPZ}, \mathrm{PZ}, \mathrm{FPZ}$ and $\mathrm{OZ}$ ) as within-subject factors. The Huynh-Feldt correction was applied to

231 analyses when two or more degrees of freedom in the numerator. Degrees of freedom are

232 reported uncorrected but it is included the epsilon value. The least significant difference (LSD)

233 test was used for post hoc multiple pairwise comparisons. Only differences that involved group

234 or any interaction by Group are reported.

235 SLORETA Analysis

236 The standardized low-resolution brain electromagnetic tomography (sLORETA) software

237 (http://www.uzh.ch/keyinst/loreta.htm), based on the scalp-recorded electric potential, was used

238 to compute the cortical three-dimensional distribution of current density of the

239 electrophysiological data during the CPT with 32-channel EEG recording, as performed in

240 previous studies (Perchet et al. 2008; Tombini et al. 2009). The sLORETA method is a three-

241 dimensionally distributed (3D), discrete, linear, minimum norm inverse solution. The sLORETA

242 standardization endows the tomography with the property of exact localization to test point

243 sources, which yields images of standardized current density with exact localization despite its

244 low spatial resolution (i.e., neighboring neuronal sources will be highly correlated). The method

245 has been described in great detail (Pascual-Marqui 2002) and the zero-error localization property

246 is described elsewhere (Pascual-Marqui, 2007).

247 Based on the current sLORETA implementation, computations were made in a realistic

248 head model (Fuchs et al. 2002) using the MNI152 template (Mazziotta et al. 2001), and with the

249 three-dimensional space solution restricted to cortical gray matter, as established in the

250 probabilistic Talairach atlas (Lancaster et al. 2000). The standard electrode positions on the

251 MNI152 scalp were taken from Jurcak et al. (2007) and Oostenveld \& Praamstra (2001). The

252 intracerebral volume is partitioned into 6239 voxels at a spatial resolution of $5 \mathrm{~mm}$, which allows 
253 the generation of images that represent the standardized electric activity at each voxel in

254 neuroanatomic Montreal Neurological Institute (MNI) space. Additionally, images are corrected

255 to Talaraich space and reported using anatomical labels, i.e., Brodmann areas (Brett et al. 2002).

256 To identify differences in current sources between groups each point was analyzed for

257 every component: P100 (between 100 and 120 ms), N150 (between 145 and 165 ms), P200

258 (between 190 and $210 \mathrm{~ms}$ ), N200 (between 230 and $290 \mathrm{~ms}$ ), and P300 (between 350 and 500

259 ms). A Statistical Non-Parametric Mapping analysis (10,000 randomizations) was performed

260 with group (skilled and novice) and conditions (target and non-target) as factors. Only the time

261 points where significant differences in the current sources were observed between groups are

262 reported. The following analyses were conducted at these time points: a) an analysis between

263 conditions (target versus non-target), separately for each group; b) an analysis between groups in

264 the target condition; and c) an analysis between groups in the non-target condition. Significant

265 differences $(\mathrm{p}<.05)$ are reported. 


\section{Results}

268

269

270

271

272

273

274

275

276

277

278

279

280

281

282

283

284

\section{Behavioral Results}

Behavioral results showed no significant differences between the two groups of athletes

for the rate of correct responses $(t(19)=0.53, p=.60)$ or false alarms $(t(19)=-0.22, p=.82)$.

Similarly, there were no differences between groups in response times $(t(19)=0.41, p=.68)($ see Table 2).

[Insert Table 2]

\section{ERP Results}

P100 and N150 were elicited mainly in occipital areas in both groups when amplitude maps were examined, while P200 was seen in central areas, N200 was distributed in centroparietal and temporal regions of the left hemisphere, and P300 is observed in the parietal region. Maximum amplitude distribution of the attention effect (i.e. target minus non-target condition) for P100 and P200 seemed to be different between groups: P100 in skilled subjects showed a left-lateral and central distribution while in novice the distribution was mainly lateralized to the right. P200 was observed in centro-parietal for skilled, meanwhile in the novice group this ERP component was centro-frontal. Nevertheless, amplitude differences between conditions in N200 were only observed in novice athletes in the centro-parietal site (see Figure 3). 
285

286

287

288

289

290

291

292

293

294

295

296

298

299

300

301

302

303

304

305

306

\section{P100: 100 to $120 \mathrm{~ms}$ Time window}

Four-way analysis of variance using lateral electrodes data (left and right regions) showed no significant differences between groups (main effect of group $F<1$ ), but a significant Condition by Group interaction was found in this time window $(F(1,19)=10.75, p=.004)$. Post hoc analyses revealed greater amplitudes elicited by target than non-target condition in the skilled athletes group $(M D=0.99 \mu \mathrm{V}, p=.005)$ than in the novice group $(M D=0.47 \mu \mathrm{V}, p=$ .15). Additionally, there was a significant Condition by Electrode site by Group interaction $(F(23,209)=2.82, p=.03$, epsilon $=0.36)$, where skilled athletes showed greater differences in amplitude between conditions, mainly at P3-P4 $(M D=1.68 \mu \mathrm{V}, p=.006), 01-\mathrm{O} 2(M D=1.68$

$\mu \mathrm{V}, p=.001), \mathrm{T} 5-\mathrm{T} 6(M D=1.36 \mu \mathrm{V}, p=.008), \mathrm{CP} 3-\mathrm{CP} 4(M D=1.33 \mu \mathrm{V}, p=.02)$ and TP7TP8 $(M D=1.14 \mu \mathrm{V}, p=.03)$. Three-way ANOVA using midline sites data showed no significant differences between groups (main effect of Group; $\mathrm{F}<1$ ) or any significant interaction by Group (All $F<1)$.

Regarding latency analyses, four-way ANOVA with lateral electrodes and three-way ANOVA with midline sites did not display significant main effects of Group (all $\mathrm{F}<1$ ) or any interaction by Group (all $F<1$ ).

\section{P200: 190 to $210 \mathrm{~ms}$ Time window}

Analysis using lateral electrodes showed no significant differences between groups (main effect of Group $(F<1)$ but does a significant Condition by Group interaction $(F(1,19)=9.97, p$ $=.005)$. Post hoc analyses revealed greater differences in amplitude between conditions (target $>$ non-target) in the skilled athletes $(\mathrm{MD}=1.39 \mu \mathrm{V}, \mathrm{p}=.008)$ compared with the novice group $(\mathrm{MD}=0.74 \mu \mathrm{V}, \mathrm{p}=.14)$. Three-way ANOVA using midline sites data showed no significant 
307 differences between groups (main effect of Group; F < 1) or any significant interaction by Group $308($ All $F<1)$.

309 Regarding latency analyses, four-way ANOVA with lateral electrodes and three-way

310 ANOVA with midline sites did not display significant main effects of Group (all F $<1$ ) or any

311 interaction by Group (all $F<1$ ).

\section{N200: 250 to $300 \mathrm{~ms}$ Time window}

Four-way ANOVA showed no significant main effect of $\operatorname{Group}(F(1,19)=1.68, p=.21)$ no significant main effect for group $(F(1,19)=2.30, p=.14)$, but there was a significant Condition by Electrode sites by Group interaction $(F(6,114)=4.25, p=.007$, epsilon $=0.54)$.

Post hoc analyses revealed differences between groups in which novices showed higher amplitudes in the target condition than the skilled group at the $\mathrm{CZ}(M D=5.12 \mu \mathrm{V}, p=.02), \mathrm{FCZ}$ $(M D=4.27 \mu \mathrm{V}, p=.04)$ and $\mathrm{CPZ}(M D=5.03 \mu \mathrm{V}, p=.02)$ electrodes. Additionally, differences in amplitude were found between conditions (target $>$ non-target) in novice athletes at the PZ $(M D=3.54 \mu \mathrm{V}, p=.02)$ and $\mathrm{CPZ}(M D=3.92 \mu \mathrm{V}, p=.009)$ electrodes, meanwhile such differences were no observed in skilled athletes. ANOVA with midline sites did not display significant main effects of Group (all $\mathrm{F}<1$ ) or any interaction by Group (all $F<1$ ). 
329 observed in novice athletes, and it looks like no differences between groups in their amplitude

330 maps within time window of the P300.

\section{sLORETA Results}

Differences in the current sources were found in at least one time point of three ERP (skilled and novice) and condition (target and non-target) as factors. No differences in P100 and N200 were observed in the current source analysis. Differences were observed at $146 \mathrm{~ms}$ in the Superior Frontal, Medial Frontal, Orbital, Rectal areas of the frontal lobe, and in the Anterior Cingulate of the limbic lobe Gyri, suggesting greater activation in these structures in skilled compared with novice athletes. When the P200 time period was analyzed, greater activation in the Anterior Cingulate of the limbic lobe was observed at $204 \mathrm{~ms}$ in skilled than in novice athletes. For the time period of the P300, skilled showed greater activation than novice athletes in different structures at three different latencies: $352 \mathrm{~ms}$ (Anterior Cingulate in the limbic lobe and Medial Frontal Gyrus in the frontal lobe), $408 \mathrm{~ms}$ (Parahippocampal Gyrus and Sub-Gyral of the limbic lobe, and Fusiform Gyrus in the temporal lobe), and 478 ms (Uncus, Parahippocampal

346 Gyrus and Anterior Cingulate of the limbic lobe, Medial Frontal Gyrus and Superior Frontal

347 Gyrus in the frontal lobe). Detailed results may be seen in Table 3, and the statistical 348 nonparametric maps are shown in Fig. 4. 
Where differences between groups were found, the following analyses were conducted: a

352

353

354

355

356

357

358

359

360

361

362

363

364

365 comparison between conditions (target versus non-target), separately for each group; a

comparison between groups in the target condition; and a comparison between groups in the non-

target condition. Significant differences between conditions were only observed in the skilled

group, while significant differences between groups were observed only in the non-target

condition. Skilled athletes showed greater activation in the target than non-target condition $(p<$

$.05)$ at 146 ms: Precuneus (right), BA 31; Sub-gyral (right), BA 31; and Cingulate Gyrus (right),

BA 31; at 352 ms: bilateral Cingulate Gyri, BA 31; bilateral Parahippocampal Gyri, BA 27;

Fusiform Gyrus (right), BA 20; bilateral Posterior Cingulate, BA 23; superior Temporal Gyrus

(right), BA 23; Insula (right), BA 13; and Sub-gyral (right), BA 21; and 408 ms: Fusiform Gyrus

(right), BA 20; and bilateral Parahippocampal Gyri, BA 36. Differences between groups at 352

ms were observed in the non-target condition $(p<.05)$ in the left Inferior Temporal Gyrus (BA

20), left Fusiform Gyrus (BA 20), left Parahippocampal Gyrus (BA 36), left Sub-gyral (BA 20),

and left Uncus (BA 20), where novice showed greater activation than skilled athletes. 
367 Discussion

368

369

370

371

372

373

374

375

376

377

378

379

380

381

382

383

384

385

386

387

The goal of this study was to investigate the differences in sustained attention between skilled and novice martial arts athletes using ERP and sLORETA as tools. Based on previous research, we expected to find better behavioral performance, larger amplitudes in attentionrelated ERP components, and greater activation in the anterior cingulate, dorsolateral prefrontal, and parietal cortical regions, primarily in the right hemisphere brain structures, in skilled than in novice athletes.

No differences in behavioral results, accuracy or response time, were observed. Since both groups showed high accuracy (almost 100\%) and no differences in response time, we can assume that our task was not highly demanding; in fact, most previous studies reported no differences in these variables between expert and less expert athletes or non-athletes. Although no differences in behavioral performance were observed, differences in brain electrical activity were found between groups. These consisted of differences in early ERP components and in activation in the anterior cingulate, frontal, and temporal structures revealed by sLORETA. Our results suggest that: a) ERP and sLORETA seem to be more sensitive tools than behavioral responses to detect differences between groups, and b) there is a different neural pattern for sustained attention in skilled athletes that is likely related to their sport expertise, and these athletes have more efficient neural mechanisms for sustained attention. stimuli in P100 and P200 than novice athletes, and there were greater amplitudes and differences in amplitude between conditions in novice than skilled athletes for the N200 component. These 
388

390

391

392

393

results suggest differences in early components related to stimulus detection, stimulus evaluation, and decision-making in attentional tasks. On the other hand, the sLORETA results indicated an activation pathway from the frontal to limbic lobe, predominantly to the right hemisphere; this is consistent with the previous reports in sustained-attention tasks that require the basal forebrain cholinergic corticopetal projection system, through direct connections primarily to a right fronto-parietal-thalamic network, for top-down processing such as in the sustained-attention task (Sarter et al. 2001). This pathway was observed more frequently during the target than during the non-target condition in skilled athletes, which may imply more uniform top-down mechanisms for sustained attention in this group.

The first differences between groups were observed around $100 \mathrm{~ms}$. In the ERP analysis, the larger amplitude in the P100 component in the skilled group could mean a different profile of brain activation modulated by expertise that is associated with spatial attention; this component has been related to the sensitivity of attention to stimulus direction (Luck 2005). In our experiment, participants were instructed to respond to arrows with a particular direction. Although the related literature has not clearly defined this component in relation to the subject skills, our results suggest a greater ability for early detection of the stimulus direction in skilled athletes compared with novices. Previous studies have indicated that acute and habitual exercise affects the early visual-evoked potentials. Neural conductivity in the visual pathway after exercise might be at least partially dependent on the individual's personal training adaptation status (Ozmerdivenli et al. 2005; Zwierko et al. 2011). These findings could be related to our results, which suggest an adaptation of the P100 component as an effect of training. After this time period, in the range of the N150 component, different structures were involved in the current source analysis. The Superior Frontal Gyrus was the earliest to show a difference 
411 between groups; in general, the superior prefrontal area, roughly coinciding with the superior

412 frontal gyrus, is the prefrontal area most consistently activated by sensory stimuli of the three

413 modalities: visual, auditory, and somatosensory (Fuster 2008). An important activation was also

414 observed in the Medial Frontal Gyrus, which is related to fundamental aspects of input-

415 processing streams (Talati \& Hirsch 2005). These findings are related to the ERP results and

416 might confirm the suggestion that skilled athletes have an earlier and enhanced ability to detect

417 stimuli.

There were amplitude differences between groups at approximately $200 \mathrm{~ms}$ in the target versus non-target comparison. The skilled athletes showed larger differences between conditions than the novices in this posterior P200 component, the nature of which remains unclear in the

421 ERP literature (Luck 2005). Some studies have associated posterior P200 with the initiation of

422

423

424

425

426

427

428

429

430

431

432

433

stimulus evaluation and decision-making (Lindholm \& Koriath 1985; Nikolaev et al. 2008; Potts

2004; Potts \& Tucker 2001). The posterior P200 response to an action-anticipation task was different between professional badminton players and non-players, with professionals showing

larger amplitudes than non-players; the authors proposed that the players showed superior actionanticipation abilities associated with an enhanced P200 effect that had a posterior-occipital distribution (Jin et al. 2011). Additionally, larger amplitudes in the P200 component have been found in sprinters compared with other populations; the authors proposed that smaller amplitudes in the control groups could indicate lower attention levels (Hamon \& Seri 1989). Based on these studies and on the hypothesis that the P200 component could be an index of a stimulusidentification process and establishing a perceptual decision (Lindholm \& Koriath 1985), this effect in our results likely reflects some generic training effects. The analysis of current sources indicates that the Anterior Cingulate was primarily activated to differentiate between groups. The 
434 activation in the Anterior Cingulate was also observed in the different time periods analyzed

435 (corresponding to P100, P200 and P300 components); a role for the Anterior Cingulate in target

436 detection and executive control has been proposed (Cabeza \& Nyberg 2000; Posner \& Petersen

437 1990; Posner et al. 1988), and it might also involve the use of information about outcome,

438 particularly reward-related outcome, to guide action selection on the basis of a cost-benefit

439 analysis, integrating information about past action outcomes to optimize voluntary choice

440 behavior (Bush et al. 2002; Hadland et al. 2003; Holroyd \& Coles 2002; Matsumoto et al. 2003;

441 Rushworth et al. 2004; Walton et al. 2006). These observations are related to our results in the

442 P200 component and the hypothesis that this component is an index of a stimulus-identification

443 process and of establishing a perceptual decision (Lindholm \& Koriath 1985), and they confirm

444 that skilled and novice athletes have different neural mechanisms for making perceptual

445 decisions.

446 Negativity in the central cortical distribution was found at approximately $200 \mathrm{~ms}$. This

447 wave is an N2b (Naatanen \& Picton 1986; Patel \& Azzam 2005). The N2b corresponds to

448 voluntary processing and is elicited when subjects selectively attend to deviations in oddball

449 paradigms (Potts et al. 1998; Sams et al. 1983). This component, which is typically evoked

450 before the motor response, has been interpreted as a reflection of stimulus identification and

451 distinction (Patel \& Azzam 2005), discrimination of a target (Senkowski \& Herrmann 2002;

452 Treisman \& Sato 1990) and response monitoring (Stroth et al. 2009). In visual discrimination

453 tasks, the N2b amplitude is directly correlated with discrimination difficulty (Senkowski \&

454 Herrmann 2002). In a previous study that investigated whether exercise and physical fitness have

455 the potential to influence electrophysiological correlates of different aspects of executive control

456 in adolescents using a go/no-go task, the authors found that in higher-fit participants, the N2 
457 amplitude was significantly reduced at the fronto-central electrodes compared with the lower-fit

458 participants; the authors suggested that physical fitness increases the efficiency of the executive

459 control system by reducing the effort required for response-monitoring processes (Stroth et al.

460 2009). Therefore, our results likely point to better executive control in skilled athletes, who do

461 not need to allocate more resources to stimulus discrimination and response monitoring, because

462 the previous stimulus identification and evaluation, and perceptual decision-making were

463 sufficient to provide a motor response.

464 The afore mentioned differences might be related to differences in the amplitude

465 topography of earlier ERP P100 and P200, which show a slightly different distribution between

466 groups along the scalp. Differences in the scalp topography but not in the source density in the

467 P100 component may be explained as a result of differences in the orientation but not in the

468 location of the dipole that results in differences in amplitude between groups since it is known

469 that amplitude of components also depends on the location and orientation of the dipole (Mosher

470 et al. 1993). On the other hand, different sensorimotor mechanisms between skilled and novice

471 athletes allocated in fronto-central and parietal brain cortex would explain differences in

472 topography and current source density in P200. Previous results support a similar argument

473 presented before (Sanchez-Lopez et al. 2014), that is to say that a difference in the pointing of

474 the oblique positive dipole gradient can produce topographical differences in the expression of

475 these components on the scalp as a result of the convergence of sensorial premotor and cognitive

476 processes (Tomberg et al. 2005).

477 No differences between groups in the amplitude of P300 were found. However, a P3b

478 component with parietal distribution was observed in both skilled and novice athletes. This P3b

479 is observed for targets that are infrequent and has been associated with attentional processing 
480 (Luck 2005). The results of the current source analysis showed differences at various points

481 along the P300 time period, with activation in frontal, limbic, and temporal structures, i.e.,

482 Medial Frontal Gyrus, Superior Frontal Gyrus, Anterior Cingulate, Parahippocampal Gyrus, Sub-

483 Gyral, Uncus, and Fusiform Gyrus. An extensive study investigating multiple brain regions

484 revealed that many cortical areas, including the superior parietal lobe (Halgren et al. 1995a), are

485 involved in P300 generation. This is likely the reason why no topographical differences between

486 groups were observed in the amplitude analysis of the P300 recorded on the scalp. Source

487 localization methods, which remove the reference effect, can increase the signal-to-noise ratio,

488 meanwhile amplitude analysis can hide tiny spatial-temporal differences, that are crucial to

489 locate different current sources within conductor volume. Given the foregoing, previous studies

490 have found that the hippocampus and superior temporal sulcus contributed to P3b generation

491 (Halgren et al. 1995b). Even frontal brain structures participated in generating P3b: the orbito-

492 frontal cortex, anterior cingulate cortex, and inferior frontal sulcus showed activation during P3

493 generation (Baudena et al. 1995). These findings are closely linked to our results showing an

494 important activation in the target condition in the skilled group that was not observed in the

495 novice group in the P300 time period. This activation might be associated with expertise, as it

496 indicates a different neural pattern for attentional control processes in skilled compared to novice

497 athletes.

498 In summary, no differences in performance were observed, but differences in amplitude

499 and source analysis of the ERP were found. We propose that brain electrical activity may

500 differentiate between skilled and novice athletes, who adopt different patterns of activity. Indeed,

501 this activity pattern has been observed in previous reports comparing expert and less expert

502 athletes or non-athletes, and it suggests superior cognitive processes in high-level athletes. Two 
main hypotheses support cognitive superiority of expert athletes in comparison with less expert athletes and non-athletes: The Neural Efficiency hypothesis (for review, see Babiloni et al. 2009; Babiloni et al. 2010a; Babiloni et al. 2010b; Del Percio et al. 2010) and the further reinterpretation, Neural Flexibility (for review, see Spinelli et al. 2011; Sanchez-Lopez et al. 2014). The Neural Efficiency hypothesis implies lower spatial cortical activation in expert athletes than less expert groups. In contrast, the Neural Flexibility hypothesis incorporates evidence that some sensorial and cognitive processes increase the recruitment of brain resources in expert athletes. According to source localization analysis results, for the P300 time span, expert athletes showed greater activation than novice athletes across different brain structures. Thus, cognitive superiority of expert athletes seems to be better supported by the

512 Neural Flexibility hypothesis. Although no behavioral advantage of experts means an important discrepancy with this hypothesis, there is a possible explanation regarding some limitations of our experimental task. The time to answer was long enough for expert athletes to delay their responses to increase their accuracy. Actually, athletes displaying their expertise must include some principles of the discipline philosophy such as controlled-impulse responses. Subjects with high rank in a combat discipline delay their answers to improve their results, i.e. a phenomenon defined by waiting longer and reacting quicker that results in slower but efficient response times (Vences de Brito and Silva, 2011). Even though non-significant reaction-time differences were found between groups, the mean reactiontime of experts was higher than that of the novices. For this reason, a lack of behavioral differences

521 should not be considered as evidence against the Neural Flexibility hypothesis.

To our knowledge, few studies have focused on athletes' attention, and none of them has desynchronization data during motor actions and during the judgment of actions (Babiloni et al. 
527 2009; Babiloni et al. 2010a; Babiloni et al. 2010b; Del Percio et al. 2010). Our study is the first

528 to clearly define the type of attention studied (i.e., sustained attention) and assess its

529 electrophysiological correlates combining two analyses (ERP and sLORETA), which confirms

530 our hypothesis and demonstrates the effect induced by sport expertise. Additionally, the

531 physiological correlates differentiating these groups, and the electrical activity and brain

532 structures involved in these processes, were characterized, and the results are consistent with the 533 findings of previous studies.

534 


\section{${ }_{536}$ Conclusions}

537 Given that skilled athletes showed larger amplitudes in early ERP components than

538 novices, it appears they could detect and make perceptual decisions about the stimulus earlier

539 than novice athletes, an early attention skill that must increase efficiency during combat sports.

540 Current source analysis located brain areas involved in sustained attention, and these areas were

541 consistent with the structures previously found to be activated (Sarter et al. 2001); however, the

542 comparisons between groups showed greater activation, mainly in frontal and limbic lobes that

543 are directly related to sustained attention, in skilled than in novice athletes. This supports the idea

544 that skilled athletes displayed higher attentional abilities than novice athletes. As a whole, this

545 study indicates differences in the neural mechanisms during controlled attention between skilled

546 and novice athletes, likely due to their differing sport expertise. 


\section{${ }_{549}$ Acknowledgments}

550 The authors are grateful for the participants' cooperation in this study. The authors also

551 acknowledge Susana A. Castro-Chavira, Leonor Casanova, Lourdes Lara, and Hector Belmont

552 for technical assistance and Dorothy Pless for revising English style. 


\section{${ }_{554}$ References}

555

556

Abernethy B, and Russell DG. 1987. Expert-Novice Differences in an Applied Selective Attention Task J Sport Exerc Psychol 9:326-345.

Anshel MH, and Payne JM. 2006. Application of Sport Psychology for Optimal Performance in Martial Arts. In: Dosil J, ed. The Sport Psychologist's Handbook A Guide for SportSpecific Performance Enhancement England: John Wiley \& Sons 353-374.

Babiloni C, Del Percio C, Rossini PM, Marzano N, Iacoboni M, Infarinato F, Lizio R, Piazza M, Pirritano M, Berlutti G, Cibelli G, and Eusebi F. 2009. Judgment of actions in experts: a high-resolution EEG study in elite athletes. Neuroimage 45:512-521. 10.1016/j.neuroimage.2008.11.035

Babiloni C, Marzano N, Iacoboni M, Infarinato F, Aschieri P, Buffo P, Cibelli G, Soricelli A, Eusebi F, and Del Percio C. 2010a. Resting state cortical rhythms in athletes: a highresolution EEG study. Brain Res Bull 81:149-156. 10.1016/j.brainresbull.2009.10.014

Babiloni C, Marzano N, Infarinato F, Iacoboni M, Rizza G, Aschieri P, Cibelli G, Soricelli A, Eusebi F, and Del Percio C. 2010b. "Neural efficiency" of experts' brain during judgment of actions: a high-resolution EEG study in elite and amateur karate athletes. Behav Brain Res 207:466-475. 10.1016/j.bbr.2009.10.034 
571 Baudena P, Halgren E, Heit G, and Clarke JM. 1995. Intracerebral potentials to rare target and

572 distractor auditory and visual stimuli. III. Frontal cortex. Electroencephalogr Clin

573 Neurophysiol 94:251-264. 10.1016/0013-4694(95)98476-O

574 Blumenstaein B, Bar-Eli M, and Tenenbaum G. 2002. Brain and Body in Sport and Exercise. 575 U.S.A: Wiley

576

577

578

Brefczynski-Lewis JA, Lutz A, Schaefer HS, Levinson DB, and Davidson RJ. 2007. Neural correlates of attentional expertise in long-term meditation practitioners. Proc Natl Acad Sci U S A 104:11483-11488. DOI 10.1073/pnas.0606552104

Brett M, Johnsrude IS, and Owen AM. 2002. The problem of functional localization in the human brain. Nat Rev Neurosci 3:243-249. 10.1038/nrn756

Bush G, Vogt BA, Holmes J, Dale AM, Greve D, Jenike MA, and Rosen BR. 2002. Dorsal anterior cingulate cortex: a role in reward-based decision making. Proc Natl Acad Sci U S A 99:523-528. 10.1073/pnas.012470999

Cabeza R, and Nyberg L. 2000. Imaging cognition II: An empirical review of 275 PET and fMRI studies. J Cogn Neurosci 12:1-47.

Cohen RM, Semple WE, Gross M, King AC, and Nordahl TE. 1992. Metabolic brain pattern of sustained auditory discrimination. Exp Brain Res 92:165-172. 
588 del-Monte L. 2005. Relación entre la capacidad de concentración de la atención y el rendimiento 589 en las judokas del Equipo Nacional de Cuba. ef deportes 87:1-1.

590 Del Percio C, Infarinato F, Iacoboni M, Marzano N, Soricelli A, Aschieri P, Eusebi F, and

591

592

593

594 595 Babiloni C. 2010. Movement-related desynchronization of alpha rhythms is lower in athletes than non-athletes: a high-resolution EEG study. Clin Neurophysiol 121:482-491. 10.1016/j.clinph.2009.12.004

Desimone R. 1996. Neural mechanisms for visual memory and their role in attention. Proc Natl Acad Sci U S A 93:13494-13499.

Fink GR, Halligan PW, Marshall JC, Frith CD, Frackowiak RS, and Dolan RJ. 1997. Neural mechanisms involved in the processing of global and local aspects of hierarchically organized visual stimuli. Brain 120 ( Pt 10):1779-1791.

Folstein MF, Folstein SE, and McHugh PR. 1975. "Mini-mental state". A practical method for grading the cognitive state of patients for the clinician. J Psychiatr Res 12:189-198. 0022$3956(75) 90026-6$

Fontani G, and Lodi L. 2002. Reactivity and event-related potentials in attentional tests: Effect of training. Percept Mot Skills 94:817-833. 
604 Fontani G, Lodi L, Felici A, Migliorini S, and Corradeschi F. 2006. Attention in athletes of high 605 and low experience engaged in different open skill sports. Percept Mot Skills 102:791606 805. Doi 10.2466/Pms.102.3.791-805

607 608

Hack J, Memmert D, and Rupp A. 2009. Attentional mechanisms in sports via brain-electrical event-related potentials. Res $Q$ Exerc Sport 80:727-738.

Fontani G, Maffei D, Cameli S, and Polidori F. 1999. Reactivity and event-related potentials during attentional tests in athletes. Eur J Appl Physiol Occup Physiol 80:308-317.

Fuchs M, Kastner J, Wagner M, Hawes S, and Ebersole JS. 2002. A standardized boundary element method volume conductor model. Clin Neurophysiol 113:702-712. S1388245702000305

Fuster MJ. 2008. The prefrontal cortex. San Diego, CA, USA: Elservier.

Gratton G, Coles MG, and Donchin E. 1983. A new method for off-line removal of ocular artifact. Electroencephalogr Clin Neurophysiol 55:468-484.

Hadland KA, Rushworth MF, Gaffan D, and Passingham RE. 2003. The anterior cingulate and reward-guided selection of actions. $J$ Neurophysiol 89:1161-1164. 10.1152/jn.00634.2002

Halgren E, Baudena P, Clarke JM, Heit G, Liegeois C, Chauvel P, and Musolino A. 1995a. Intracerebral potentials to rare target and distractor auditory and visual stimuli. I. Superior 
temporal plane and parietal lobe. Electroencephalogr Clin Neurophysiol 94:191-220.

622 $001346949400259 \mathrm{~N}$

623

624

625

626

627

628

Halgren E, Baudena P, Clarke JM, Heit G, Marinkovic K, Devaux B, Vignal JP, and Biraben A. 1995b. Intracerebral potentials to rare target and distractor auditory and visual stimuli. II. Medial, lateral and posterior temporal lobe. Electroencephalogr Clin Neurophysiol $94: 229-250.001346949598475 \mathrm{~N}$

Hamon JF, and Seri B. 1989. Cortical reactivity during reaction time tests in sprinters. Neurophysiol Clin 19:109-122.

Holroyd CB, and Coles MG. 2002. The neural basis of human error processing: reinforcement learning, dopamine, and the error-related negativity. Psychol Rev 109:679-709.

Hopfinger JB, Buonocore MH, and Mangun GR. 2000. The neural mechanisms of top-down attentional control. Nat Neurosci 3:284-291.

Hung T-M, Spalding TW, Santa María DL, and Hatfield BD. 2004. Assessment of Reactive Motor Performance With Event-Related Brain Potentials: Attention Processes in Elite Table Tennis Players. J Sport Exerc Psychol 26.

Jennings JR, and Coles MGH. 1991. Handbook of cognitive psychophysiology: Central and autonomic nervous system approaches. Chichester, UK: Wiley. 
638 Jin H, Xu G, Zhang JX, Gao H, Ye Z, Wang P, Lin H, Mo L, and Lin CD. 2011. Event-related

639

640

641

642

643

644

645

646

647

648

649

650

651

652

653

654 potential effects of superior action anticipation in professional badminton players. Neurosci Lett 492:139-144. S0304-3940(11)00120-0

Jurcak V, Tsuzuki D, and Dan I. 2007. 10/20, 10/10, and 10/5 systems revisited: their validity as relative head-surface-based positioning systems. Neuroimage 34:1600-1611.

Lancaster JL, Woldorff MG, Parsons LM, Liotti M, Freitas CS, Rainey L, Kochunov PV, Nickerson D, Mikiten SA, and Fox PT. 2000. Automated Talairach atlas labels for functional brain mapping. Hum Brain Mapp 10:120-131. 10.1002/10970193(200007)10:3<120::AID-HBM30>3.0.CO;2-8

Lane RD, Chua PM, and Dolan RJ. 1999. Common effects of emotional valence, arousal and attention on neural activation during visual processing of pictures. Neuropsychologia 37:989-997. S0028393299000172

Lavalle D, Kremer J, Moran AP, and Williams M. 2004. Sport Psychology. Contemporany Themes. London: Palgrave Macmillan.

Lindholm E, and Koriath JJ. 1985. Analysis of multiple event related potential components in a tone discrimination task. Int J Psychophysiol 3:121-129. 
655 Matsumoto K, Suzuki W, and Tanaka K. 2003. Neuronal correlates of goal-based motor 656 selection in the prefrontal cortex. Science 301:229-232. 10.1126/science.1084204

657

658

659

660

661

662

663

664

665
Mazziotta J, Toga A, Evans A, Fox P, Lancaster J, Zilles K, Woods R, Paus T, Simpson G, Pike B, Holmes C, Collins L, Thompson P, MacDonald D, Iacoboni M, Schormann T, Amunts K, Palomero-Gallagher N, Geyer S, Parsons L, Narr K, Kabani N, Goualher GL, Boomsma D, Cannon T, Kawashima R, and Mazoyer B. 2001. A probabilistic atlas and reference system for the human brain: International Consortium for Brain Mapping (ICBM). Philos Trans R Soc Lond B Biol Sci 356:1293-1322. 10.1098/rstb.2001.0915

Mosher JC, Spencer ME, Leahy RM, and Lewis PS. 1993. Error bounds for EEG and MEG dipole source localization. Electroencephalography and Clinical Neurophysiology 86:303-321. http://dx.doi.org/10.1016/0013-4694(93)90043-U

Naatanen R, and Picton TW. 1986. N2 and automatic versus controlled processes. Electroencephalogr Clin Neurophysiol Suppl 38:169-186.

Nikolaev AR, Ziessler M, Dimova K, and van Leeuwen C. 2008. Anticipated action consequences as a nexus between action and perception: evidence from event-related potentials. Biol Psychol 78:53-65. S0301-0511(08)00003-3

Nougier V, and Rossi B. 1999. The development of expertise in the orientation of attention Int J Sport Psychol 30:246-260. 
673 Oostenveld R, and Praamstra P. 2001. The five percent electrode system for high-resolution EEG 674 and ERP measurements. Clin Neurophysiol 112:713-719. S1388-2457(00)00527-7

675 Ozmerdivenli R, Bulut S, Bayar H, Karacabey K, Ciloglu F, Peker I, and Tan U. 2005. Effects of 676 exercise on visual evoked potentials. Int J Neurosci 115:1043-1050.

677 N575HU55NMT73306

678

679

680

681

682

683

684

685

686
Pardo JV, Fox PT, and Raichle ME. 1991. Localization of a human system for sustained attention by positron emission tomography. Nature 349:61-64. 10.1038/349061a0

Pascual-Marqui RD. 2002. Standardized low-resolution brain electromagnetic tomography (sLORETA): technical details. Methods Find Exp Clin Pharmacol 24 Suppl D:5-12.

Pascual-Marqui RD. October 17, 2007. Discrete, 3D Distributed, Linear Imaging Methods of Electric Neuronal Activity. Part 1: Exact, Zero Error Localization. arXiv:07103341 [math-ph].

Patel SH, and Azzam PN. 2005. Characterization of N200 and P300: selected studies of the Event-Related Potential. Int J Med Sci 2:147-154.

Perchet C, Godinho F, Mazza S, Frot M, Legrain V, Magnin M, and Garcia-Larrea L. 2008. Evoked potentials to nociceptive stimuli delivered by $\mathrm{CO} 2$ or Nd:YAP lasers. Clin Neurophysiol 119:2615-2622. 10.1016/j.clinph.2008.06.021 
690 Picton TW, Bentin S, Berg P, Donchin E, Hillyard SA, Johnson R, Jr., Miller GA, Ritter W, 691 Ruchkin DS, Rugg MD, and Taylor MJ. 2000. Guidelines for using human event-related 692 potentials to study cognition: recording standards and publication criteria.

693 Psychophysiology 37:127-152.

694 Posner MI, and Petersen SE. 1990. The attention system of the human brain. Annu Rev Neurosci 695 13:25-42. 10.1146/annurev.ne.13.030190.000325

696

697

698

699

700

701

702

703

704

705

706

707

Posner MI, Petersen SE, Fox PT, and Raichle ME. 1988. Localization of cognitive operations in the human brain. Science 240:1627-1631.

Potts GF. 2004. An ERP index of task relevance evaluation of visual stimuli. Brain Cogn 56:513. $10.1016 /$ j.bandc. 2004.03 .006

Potts GF, Dien J, Hartry-Speiser AL, McDougal LM, and Tucker DM. 1998. Dense sensor array topography of the event-related potential to task-relevant auditory stimuli. Electroencephalogr Clin Neurophysiol 106:444-456. S0013469497001600 [pii]

Potts GF, and Tucker DM. 2001. Frontal evaluation and posterior representation in target detection. Brain Res Cogn Brain Res 11:147-156. S0926-6410(00)00075-6

Radlo SJ, Janelle CM, Barba DA, and Frehlich SG. 2001. Perceptual decision making for baseball pitch recognition: Using P300 latency and amplitude to index attentional processing. Research Quarterly for Exercise and Sport 72:22-31. 
708 Riccio CA, Reynolds CR, Lowe P, and Moore JJ. 2002. The continuous performance test: a 709 window on the neural substrates for attention? Archives of Clinical Neuropsychology 17:235-272. http://dx.doi.org/10.1016/S0887-6177(01)00111-1

711 Rushall BR. 2006. Psychological Factors and Mental Skills in Wrestling. In: Dosil J, ed. The Sport Psychologist's Handbook A Guide for Sport-Specific Performance Enhancement. Inglaterra: John Wiley \& Sons

714

715

716

717

718

719

720

721
Rushworth MF, Walton ME, Kennerley SW, and Bannerman DM. 2004. Action sets and decisions in the medial frontal cortex. Trends Cogn Sci 8:410-417. 10.1016/j.tics.2004.07.009

Sams M, Alho K, and Naatanen R. 1983. Sequential effects on the ERP in discriminating two stimuli. Biol Psychol 17:41-58. 0301-0511(83)90065-0

Sánchez-López J, Fernández T, Silva-Pereyra J, and Martínez-Mesa JA. 2013. Differences between Judo, Taekwondo and Kung-fu Athletes in Sustained Attention and Impulse Control. Psychology 4:607-612. DOI:10.4236/psych.2013.47086

Sánchez-López J, Fernández T, Silva-Pereyra J, Martínez-Mesa JA, and Moreno-Aguirre AJ. 2014. Evaluación de la Atención en Deportistas de Artes Marciales. Expertos vs. Novatos [Measurement of Attention in Martial Arts Athletes.Skilled vs. Novices] Revista de Psicología del Deporte 23:87-94. 
726 Sanchez-Lopez J, Fernandez T, Silva-Pereyra J, Martinez Mesa JA, and Di Russo F. 2014.

727 Differences in visuo-motor control in skilled vs. novice martial arts athletes during sustained and transient attention tasks: a motor-related cortical potential study. PLoS One

729 9:e91112. 10.1371/journal.pone.0091112

730

731

732

Sarter M, Givens B, and Bruno JP. 2001. The cognitive neuroscience of sustained attention: where top-down meets bottom-up. Brain Res Brain Res Rev 35:146-160. S0165017301000443

Senkowski D, and Herrmann CS. 2002. Effects of task difficulty on evoked gamma activity and ERPs in a visual discrimination task. Clin Neurophysiol 113:1742-1753. S1388245702002663

Smid HG, de Witte MR, Homminga I, and van den Bosch RJ. 2006. Sustained and transient attention in the continuous performance task. J Clin Exp Neuropsychol 28:859-883. NQ3056740652R305

Spinelli D, DiRusso F, and Pitzalis S. 2011. Il Cervello dell'Atleta [The Athlete's Brain] In: F L, ed. Sportivamente: Temi di Psicologia dello Sport [Sportivamente: Sport Psichology Topics]. Roma: LED, 109-140.

Stroth S, Kubesch S, Dieterle K, Ruchsow M, Heim R, and Kiefer M. 2009. Physical fitness, but not acute exercise modulates event-related potential indices for executive control in healthy adolescents. Brain Res 1269:114-124. 10.1016/j.brainres.2009.02.073 
745 Talati A, and Hirsch J. 2005. Functional Specialization within the Medial Frontal Gyrus for

746 Perceptual Go/No-Go Decisions Based on "What," "When," and "Where" Related

747 Information: An fMRI Study. J Cogn Neurosci 17:981-993. 10.1162/0898929054475226

748

749
Tang YY, and Posner MI. 2009. Attention training and attention state training. Trends Cogn Sci 13:222-227. 10.1016/j.tics.2009.01.009

Thompson T, Steffert T, Ros T, Leach J, and Gruzelier J. 2008. EEG applications for sport and performance. Methods 45:279-288. S1046-2023(08)00116-3

Tomberg C, Weinberg H, Vrba J, and Tcheung T. 2005. Paradoxical scalp lateralization of the P100 cognitive somatic potential in humans: a magnetic field study. Neurosci Lett 391:68-70.

Tombini M, Zappasodi F, Zollo L, Pellegrino G, Cavallo G, Tecchio F, Guglielmelli E, and Rossini PM. 2009. Brain activity preceding a 2D manual catching task. Neuroimage 47:1735-1746. 10.1016/j.neuroimage.2009.04.046

Treisman A, and Sato S. 1990. Conjunction search revisited. J Exp Psychol Hum Percept Perform 16:459-478.

Vences de Brito A, and Silva C. 2011. Reaction Time in Karate Athletes. IDO MOVEMENT FOR CULTURE Journal of Martial Arts Anthropology 11:35-39. 
762 Walton ME, Kennerley SW, Bannerman DM, Phillips PE, and Rushworth MF. 2006. Weighing

763

764

765

766

767

768

769 up the benefits of work: behavioral and neural analyses of effort-related decision making. Neural Netw 19:1302-1314. 10.1016/j.neunet.2006.03.005

Williams AM, and Grant A. 1999. Training perceptual skill in sport. Int J Sport Psychol 30:194220.

Zwierko T, Lubinski W, Lubkowska A, Niechwiej-Szwedo E, and Czepita D. 2011. The effect of progressively increased physical efforts on visual evoked potentials in volleyball players and non-athletes. J Sports Sci 29:1563-1572. 10.1080/02640414.2011.605166 
Table $\mathbf{1}$ (on next page)

Participants characteristics

Participants characteristics 
1 Table 1

\begin{tabular}{|c|c|c|c|c|c|}
\hline Status & Age & $\begin{array}{c}\text { Year of Sport } \\
\text { Practice }\end{array}$ & $\begin{array}{c}\text { Intelligence } \\
\text { IQ }\end{array}$ & $\begin{array}{l}\text { ADHD } \\
\text { score }\end{array}$ & Sport \\
\hline Skilled & $M=25.4$ & $M=9.4$ & $M=102$ & $M=1.79$ & Judo $=6$ \\
\hline \multirow[t]{3}{*}{$N=11$} & $S D=11.5$ & $S D=6.14$ & $S D=8$ & $S D=1.93$ & \\
\hline & & & & & $\mathrm{TKD}=4$ \\
\hline & & & & & KungFu $=1$ \\
\hline Novice & $M=25.5$ & $M=1$ & $M=107$ & $M=1$ & Judo $=3$ \\
\hline \multirow[t]{3}{*}{$N=10$} & $S D=9.05$ & $S D=0$ & $S D=6$ & $S D=1.65$ & \\
\hline & & & & & $\mathrm{TKD}=2$ \\
\hline & & & & & KungFu $=5$ \\
\hline $\begin{array}{l}\text { Skilled vs. } \\
\text { Novice }\end{array}$ & NS & $p=.001 * *$ & NS & NS & NS \\
\hline
\end{tabular}

$3 M$ =mean; $S D=$ standard deviation; TKD = tae-kwon-do; NS = No significant differences 4 


\section{Table 2 (on next page)}

Behavioral results for CPT

Behavioral results for CPT: rates of hits, false alarms, and response times for both skilled and novice groups 
$1 \quad$ Table 2

\begin{tabular}{llll}
\hline & & Skilled & Novice \\
\hline CPT & Hit rate (\%) & Mean $=97.2 \pm 2.6$ & Mean $=97.8 \pm 1.6$ \\
& False Alarms rate (\%) & Mean $=0.5 \pm 0.3$ & Mean $=0.6 \pm 0.4$ \\
& Response Time (ms) & Median $=409.8 \pm 48.0$ & Median $=400.0 \pm 59.0$
\end{tabular}

2 


\section{Table 3(on next page)}

sLORETA results for latencies, structures, localization, and statistical values.

sLORETA results for latencies, structures, localization, and statistical values. Greater differences between conditions were observed in skilled compared to novice athletes. 
1 Table 3

\begin{tabular}{|c|c|c|c|c|c|c|c|c|c|}
\hline \multicolumn{10}{|c|}{ Skilled $>$ Novice } \\
\hline \multirow{2}{*}{$\begin{array}{l}\text { Latency } \\
\text { (ms) }\end{array}$} & \multicolumn{3}{|c|}{ TAL } & \multirow[t]{2}{*}{ Brodmann } & \multirow[t]{2}{*}{ Structure } & \multirow[t]{2}{*}{ Cluster } & \multirow[t]{2}{*}{ Hemisphere } & \multirow[t]{2}{*}{ Value } & \multirow[t]{2}{*}{$p$} \\
\hline & $\mathrm{X}$ & $\mathrm{Y}$ & $\mathrm{Z}$ & & & & & & \\
\hline \multirow[t]{6}{*}{146} & 25 & 59 & 15 & 10 & Superior Frontal Gyrus (FL) & & Right & 4.55 & $<.05$ \\
\hline & 15 & 49 & 7 & 10 & Medial Frontal Gyrus (FL) & 2 & Right & 4.53 & $<.05$ \\
\hline & 10 & 44 & 7 & 32 & Anterior Cingulate (LL) & 2 & Right & 4.52 & $<.05$ \\
\hline & -5 & 48 & -19 & 11 & Orbital Gyrs (FL) & & Left & 4.45 & $<.05$ \\
\hline & -5 & 52 & -24 & 11 & Rectal Gyrus (FL) & & Left & 4.45 & $<.05$ \\
\hline & -5 & 52 & -19 & 11 & Superior Frontal Gyrus (FL) & & Left & 4.44 & $<.05$ \\
\hline 204 & 5 & 34 & -6 & 32 & Anterior Cingulate (LL) & & Right & 4.43 & $<.05$ \\
\hline \multirow[t]{3}{*}{352} & 5 & 35 & 12 & 32 & Anterior Cingulate (LL) & 9 & Right & 5.19 & $<.01$ \\
\hline & 10 & 40 & 16 & 9 & Medial Frontal Gyrus (FL) & & Right & 4.70 & $<.05$ \\
\hline & 10 & 34 & 7 & 24 & Anterior Cingulate (LL) & 3 & Right & 4.52 & $<.05$ \\
\hline \multirow[t]{5}{*}{408} & -15 & -39 & -6 & 30 & Parahippocampal Gyrus (LL) & & Left & 4.81 & $<.05$ \\
\hline & -20 & -39 & -6 & 36 & Parahippocampal Gyrus (LL) & 2 & Left & 4.55 & $<.05$ \\
\hline & -15 & -44 & -6 & 19 & Sub-Gyral (LL) & & Left & 4.48 & $<.05$ \\
\hline & -20 & -44 & -6 & 16 & Parahippocampal Gyrus (LL) & & Left & 4.37 & $<.05$ \\
\hline & -25 & -40 & -15 & 37 & Fusiform Gyrus (TL) & & Left & 4.35 & $<.05$ \\
\hline \multirow[t]{8}{*}{478} & -20 & -11 & -29 & 28 & Uncus (LL) & 7 & Left & 5.29 & $<.05$ \\
\hline & -20 & -6 & -29 & 36 & Uncus (LL) & 4 & Left & 5.13 & $<.05$ \\
\hline & 0 & 54 & 7 & 10 & Medial Frontal Gyrus (FL) & 38 & Medial & 4.62 & $<.05$ \\
\hline & -20 & -11 & -25 & 35 & Parahippocampal Gyrus (LL) & 2 & Left & 4.60 & $<.05$ \\
\hline & -5 & 58 & -3 & 10 & Superior Frontal Gyrus (FL) & 6 & Left & 4.39 & $<.05$ \\
\hline & 5 & 54 & 16 & 9 & Medial Frontal Gyrus (FL) & & Right & 4.29 & $<.05$ \\
\hline & 0 & 48 & -2 & 32 & Anterior Cingulate (LL) & 4 & Medial & 4.26 & $<.05$ \\
\hline & -15 & -6 & -21 & 34 & Uncus (LL) & & Left & 4.21 & $<.05$ \\
\hline
\end{tabular}

$2 \mathrm{FL}=$ Frontal Lobe, $\mathrm{LL}=$ Limbic Lobe, $\mathrm{TL}=$ Temporal Lobe. 
1

Continuous Performance Task.

Fig 1 - Continuous Performance Task. Type, conditions, and probability of stimuli used in CPT.

\section{Continuous Performance Task}

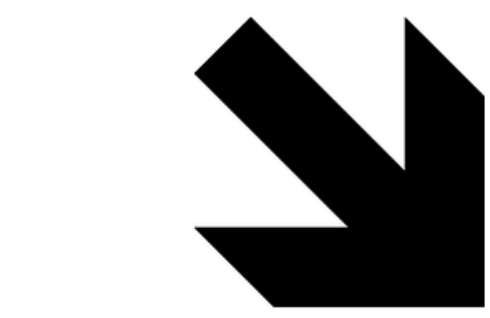

Target $=20 \%$
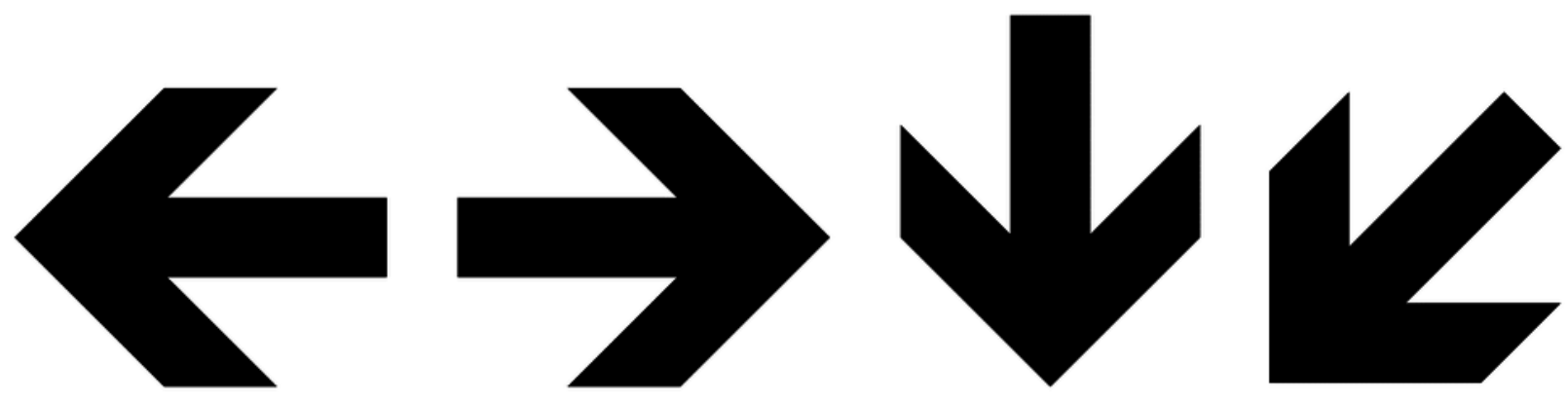

non-Target $=80 \%$ 
2

Event-related potential waves

Fig 2 - Event-related potential waves. ERP grand averages of both target (continuous lines) and non-target (dotted lines) conditions across posterior electrodes. Negative voltage is plotted upward. Black lines represent skilled athletes, and gray lines represent novice athletes. Time windows analyzed in which significant differences were found are shaded gray.

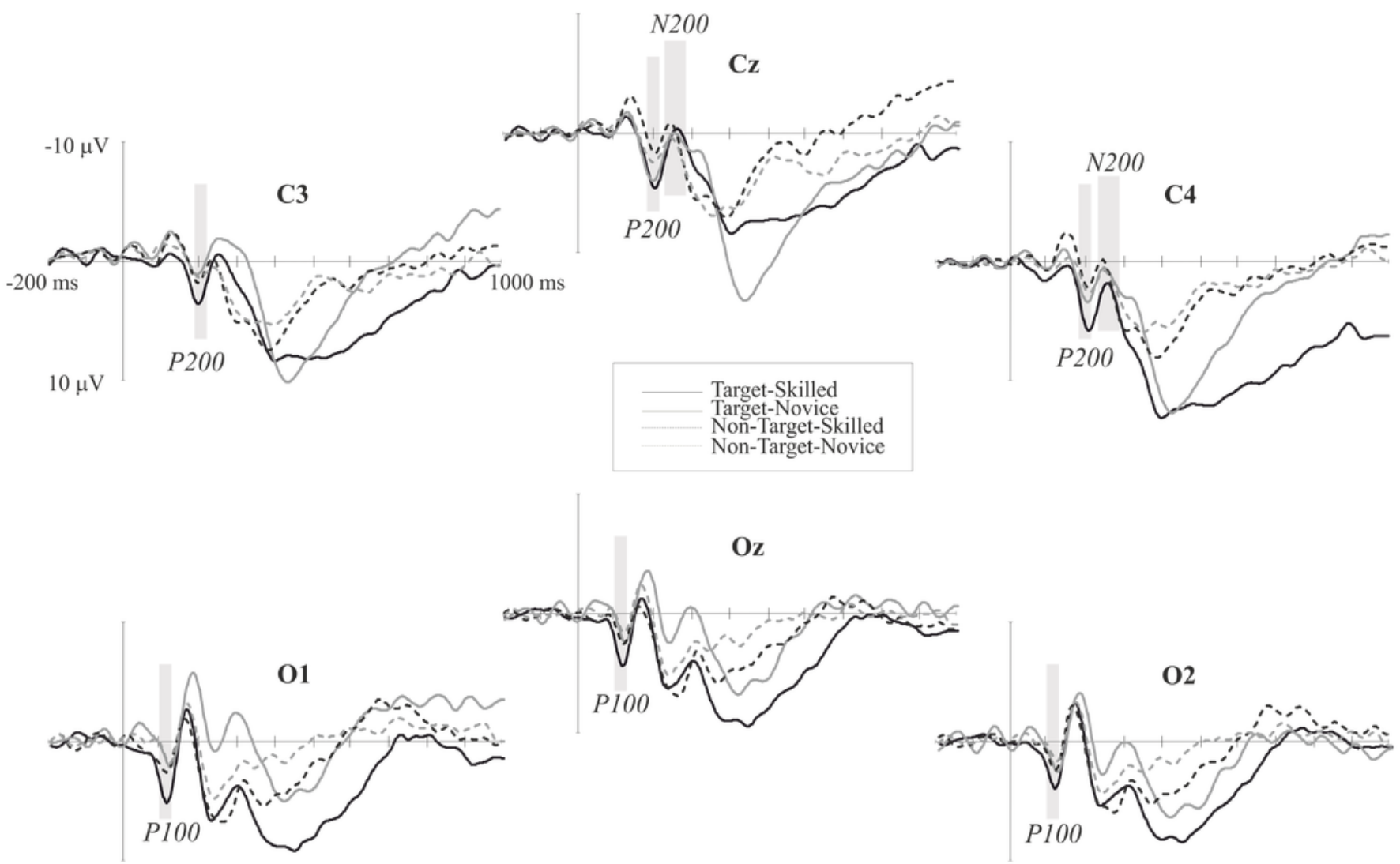




\section{3}

Event-related potentials topography.

Fig 3 - Event-related potentials topography. CPT task scalp maps showing representations of the mean amplitudes analyzed in the time windows of the target and nontarget conditions and amplitude differences. Skilled athletes are on the left side, and novice athletes are on the right side. Higher P100 and P200 response amplitudes were found in skilled athletes. P300 is also represented, but no significant differences between groups were found. 


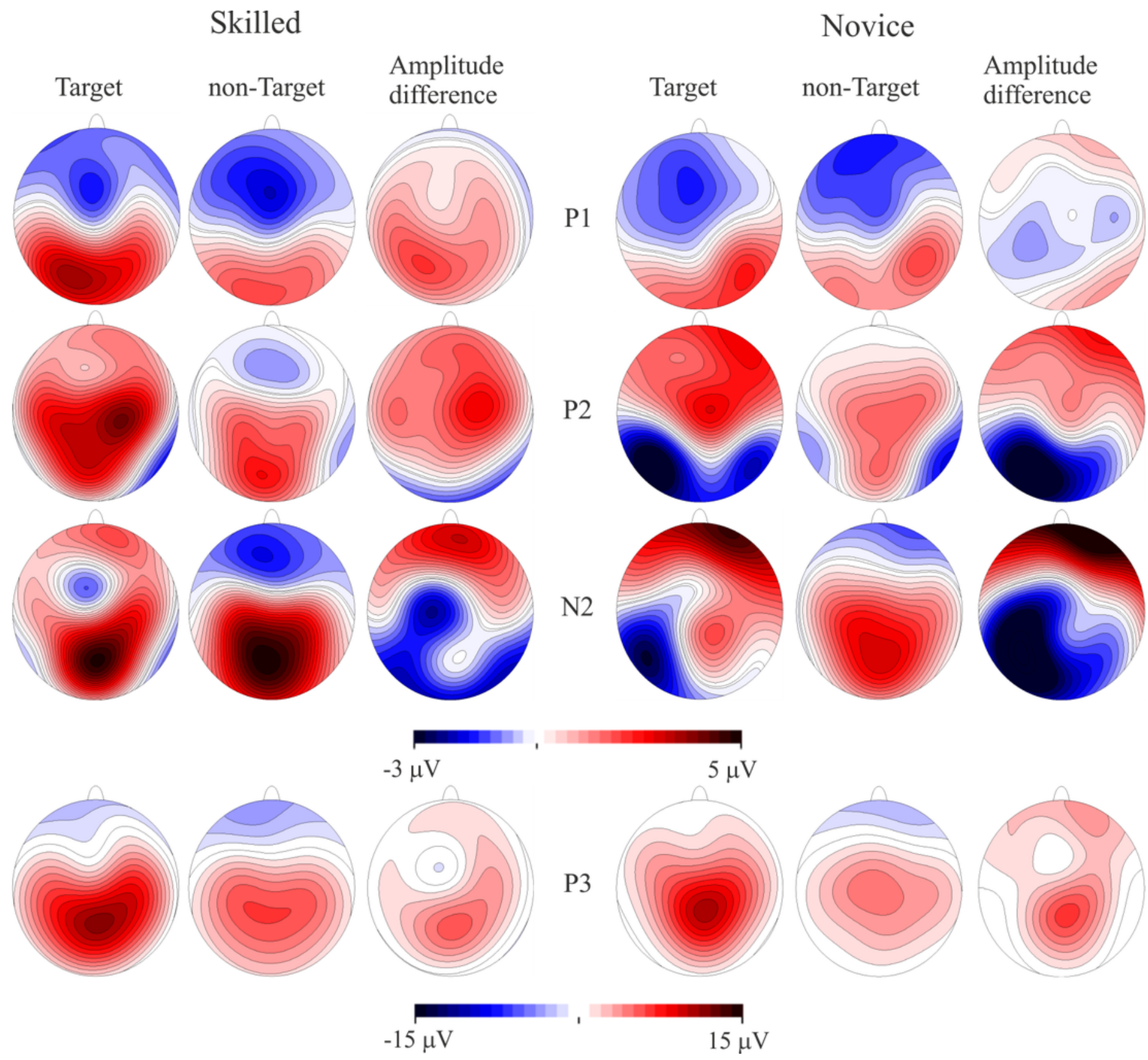


4

Current source maps.

Fig 4 - Current source maps. Differences are shown at different time points for each component where significant differences were observed: P100 at 146 ms, P200 at 204 ms, and P300 at 352 ms, 408 ms, and 478 ms.Calibration bars indicate $t$-values. Colored areas (red and blue) represent significant values $p<.05$. Positive values mean higher condition differences (target > non-target) in skilled compared with novice athletes, while negative values mean higher condition differences (target $>$ non-target) in novice compared with skilled athletes; the results only showed higher differences in skilled compared to the novice group.

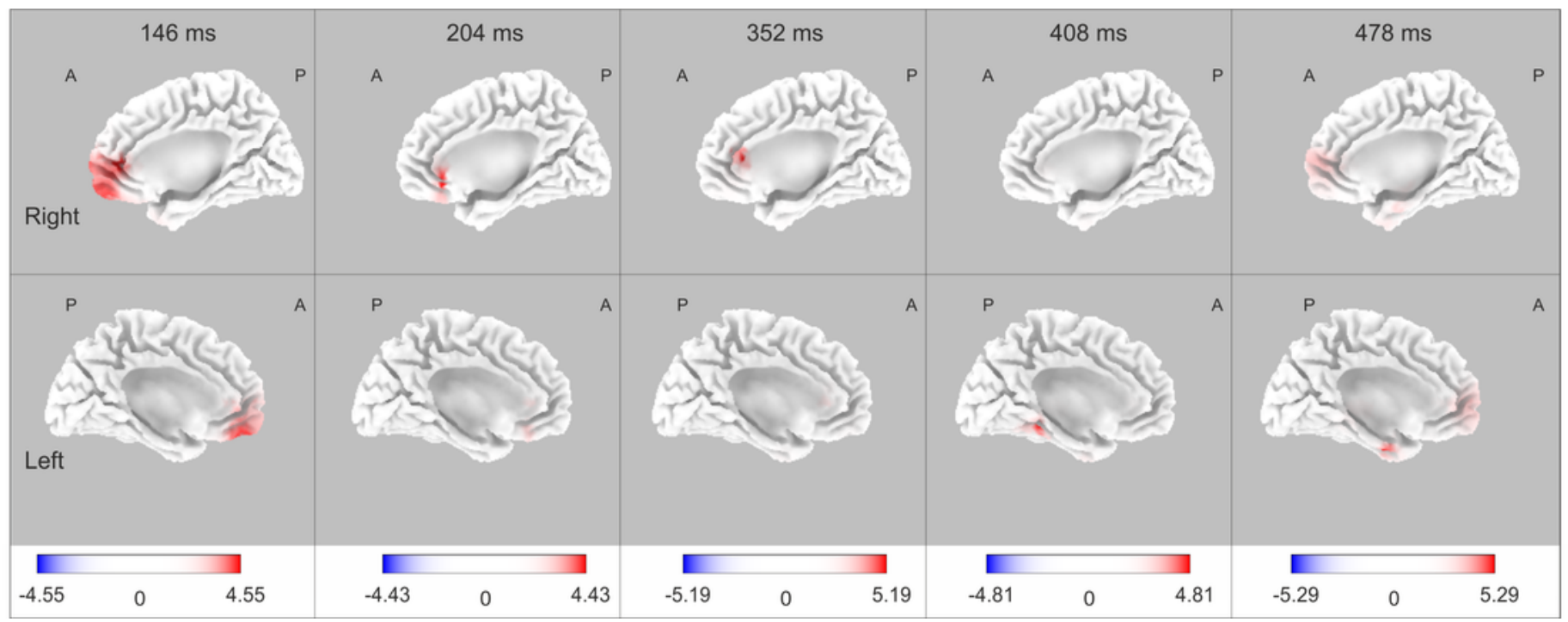

